\title{
Morphometric analysis of vastus medialis oblique muscle and its influence on anterior knee pain
}

\author{
Marwa M El Sawy ${ }^{1}$, Dalia M E EL Mikkawy ${ }^{2}$, Sayed M El-Sayed ${ }^{1}$, Ahmed M. Desouky ${ }^{1}$ \\ ${ }^{1}$ Department of Anatomy, Faculty of Medicine, Ain Shams University, Cairo, ${ }^{2}$ Department of Physical Medicine, Rheumatology and Rehabilitation, \\ Faculty of Medicine, Ain Shams University, Cairo, Egypt
}

\begin{abstract}
Healthy knees require full range squatting movements. Vastus medialis (VM) muscle regulates and adjusts the extensor apparatus that influences the patellofemoral function. This work was designed to investigate the anatomy and morphometry of vastus medialis oblique (VMO) muscle by widely used imaging techniques and investigate how VMO muscle participates in anterior knee pain. Ten dissected cadaveric specimens were examined, focusing on fiber orientations, origin, insertions and nerve supply of VMO muscle. Magnetic resonance imaging and ultrasound of VMO muscle were recorded. Anatomical cross-sectional areas of VMO muscle were determined in painless and painful knees and statistically analyzed. In cadaveric specimens, there was distinct separation between VM longus and VMO (change in fiber angle or fibrofascial plane). VMO inserted directly into the medial proximal margin of the patella, capsule of the knee joint and continuous with the patellar tendon. Separate branch of femoral nerve run along the anteromedial border of the muscle. Anatomical cross-sectional area was significantly decreased in painful knee by $-17.2 \% \pm 11.0 \%$ at lower end of shaft of femur, $-21.1 \% \pm 6.0 \%$ at upper border of patella, $-36.7 \% \pm 11.0 \%$ at mid-patellar level. VMO is distinct muscle within quadriceps femoris group. VMO muscle would track the patella medially and participate in last phase of knee extension. Assessment of the VMO muscle anatomical cross-sectional area by ultrasonography may constitute promising and reliable tool to evaluate patellofemoral pain syndrome staging.
\end{abstract}

Key words: Vastus medialis, Measures, Anterior knee pain

Received September 28, 2020; Revised November 1, 2020; Accepted November 2, 2020

\section{Introduction}

People subjected to excessive impact of physical effort at work are susceptible to knee damages while others complain of anterior knee pain due to conditions like arthritis or simple wear-and-tear with age [1]. Patellofemoral pain syndrome

\section{Corresponding author:}

Marwa M El Sawy (iD

Department of Anatomy, Faculty of Medicine, Ain Shams University, Cairo 11566, Egypt

E-mail: drmarwaelsawy@gmail.com
(PFPS) is one of the most frequent causes of anterior knee pain in adolescents and adults $[2,3]$. This illness can much affect patients' capability and quality of life and walk $[4,5]$. PFPS is often caused by imbalances in the muscles surrounding the knee, which affect the kneecap (patella) and cartilage within the joint. This syndrome most frequently caused by quadriceps weakness or tight lateral structures such as iliotibial band and vastus lateralis (VL). PFPS sometimes gets better on its own without any treatment, though you may have symptoms for several years [6].

Clinicians know that injuries to a single part of the musculoskeletal system necessarily impinge on the workings of other (even remotely distant) parts. Thus, weak quadriceps

\section{Copyright ( ) 2021. Anatomy \& Cell Biology}

This is an Open Access article distributed under the terms of the Creative Commons Attribution Non-Commercial License (http://creativecommons.org/licenses/by-nc/4.0/) which permits unrestricted non-commercial use, distribution, and reproduction in any medium, provided the original work is properly cited. 
can lead to an unstable knee joint. And an unstable knee joint can lead to weak quadriceps. It's a vicious cycle [7]. Such information would be clinically important because it could improve the design of rehabilitation protocols [8].

The most distal fibers of vastus medialis (VM) are usually described as a separate part of the muscle, the vastus medialis oblique (VMO) muscle. The primary function of the VMO was medial stabilization of the patella throughout extension. This view has been supported by several authors [9-11]. The quadriceps heads (specifically the VM) are suspected to play a role in pain and degenerative changes in the femoropatellar and femorotibial joints [10]. Painful knees frequently had $5.2 \%$ lower quadriceps muscle cross-sectional areas than contralateral pain-free knees, whereas thigh flexor and adductor muscles anatomical cross-sectional area (ACSA) didn't differ significantly between painful and painfree knees [12]. Numerous studies have examined the connection amongst muscle strength, structural pathology (e.g. radiographic stage, cartilage loss) and knee symptoms (pain and physical function) [12, 13]. Villafañe et al. [14] found that muscle strengthening addressed to the symptomatic knee reduced pain.

Magnetic resonance imaging (MRI) of skeletal muscle, allowed investigators to accurately assess muscle mass at an individual time point and its changes over time. However, usage of ultrasound (U/S) has been promoted as a possibly reliable means for the measurement of skeletal muscle mass in young and older healthy volunteers [15] and in experimental populations, such as intensive care patients [16]. Imaging procedures such as computed tomography (CT) and MRI are expensive means and are not available to the majority of clinicians and researchers. Nevertheless, the literature has not established consensus on the 'best' technique to measure cross sectional muscle area. Because of the need for consensus and standardization for both clinicians and researchers, VMO was investigated in anatomical and morphometric aspects by widely used imaging techniques and was investigated for how VMO participates in anterior knee pain. Within this broader context, we focused on the challenge of early detection of knee problems through radiological measurements of VMO.

\section{Materials and Methods}

\section{Cadaveric study}

Morphological investigations were performed on ten ca- daveric lower limbs, which were provided by the Department of Anatomy, Ain Shams University following the ethical guidelines "On the use of cadavers and parts of cadavers in medical research and for pre-, postgraduate and continued education and research with human subjects". Ten cadaveric specimens (fresh or formalin-based cadaver) were examined. The cadaver parts were investigated using macro-dissection techniques based on a standardized dissection protocol. Each lower limb was placed supine on the dissection table. The femoral nerve and artery were localized and traced distally. With the aid of these neurovascular structures, the rectus femoris, VL, vastus intermedius, and VM were identified. $\mathrm{VM}$ was traced from proximal to distal till its insertion. The different parts of VM were studied with special emphasis on the direction of the muscle fibers [17].

\section{Participant characteristics}

Forty subjects were selected by authors for the present study and divided into two groups. Group (I) (control group); 20 young, healthy males (age $=24 \pm 3$ years, body mass index $[\mathrm{BMI}]=24.1 \pm 2.7 \mathrm{~kg} / \mathrm{m}^{2}$ ) with no any previous anterior knee pain, trauma, surgery, or other lower extremity disease. The subjects in this group were recruited from the staff and students from Faculty of Medicine, Ain Shams University. Group (II) (study group); 20 males recruited in Ain Shams University Hospital, from 20 to 24 years old (BMI=25.1 \pm 1.8 $\mathrm{kg} / \mathrm{m}^{2}$ ) who presented unilateral PFPS. They were recruited from the waiting list in Rheumatology and Rehabilitation Department. The initial diagnosis was established combining medical records by CT or MRI and the presence of anterior knee pain $[14,18]$. Patients were included in this study if the following criteria were fulfilled: onset of pain longer than 3 months; positive clinical signs of PFPS (i.e., retro patellar pain, crepitation, pain in patellar grinding, direct patellar compression); no history of physical therapy. The exclusion criteria were previous knee surgery, previous meniscal or ligamentous injuries, or musculoskeletal disorders. Subjects with a lower limbs dissymmetry, with cardiac or vision dysfunctions, or neurologic (whether central or peripheral) which can influence quadriceps circumference, were also excluded from the present study [14]. All participants were subjected to MRI and U/S studies of the anterior compartment of the thigh with all experimental procedures conducted in the physical therapy research lab of the hospital from 2017 to 2019 (Retrospective study). Ethical approval for the present study was obtained from the Ethics Committee of Ain 
Shams University, Faculty of medicine institutional research board (ASU-IRB).

\section{Anatomical Cross-Sectional Area assessments}

\section{Magnetic resonance imaging measurements}

By means of a MRI scanner (Signa 1.5T; GE Healthcare, Madison, WI, USA), a sequence of axial, sagittal and coronal MRI pictures (echo time: $10 \mathrm{~ms}$, repetition time: $520 \mathrm{~ms}$, matrix: $256 \times 192$, field of view: $24 \mathrm{~cm}$, slice thickness: $1 \mathrm{~cm}$ ) of the entire right thigh was attained after the subject had been lying supine for minimum 20 minutes [19]. The subjects lay supine with their legs completely extended and muscles relaxed throughout MRI. From the MRI pictures, the outlines of VMO were manually digitized, and ACSA of the muscles were determined using ImageJ2 software [20]. Attention was done to eliminate observable intermuscular adipose and connective tissue. Each image was digitized two times, and the mean values were used for further analysis.

\section{Ultrasonography}

ACSA was assessed via the same investigator from images obtained in vivo at rest using US images recorded by a Mindray Z6 Digital Ultrasonic Diagnostic System (Shenzhen Mindray Bio-Medical Electronics Co., Ltd., Shenzhen, China) by using a linear transducer type L4-P with a frequency bandwidth range of 5-10 MHz. ACSA has been assessed by placing the U/S probe transversally relative to the patella and evaluated as the perpendicular space between the skeletal muscle interfaces [21]. The transducer was located on the anteromedial thigh along the sagittal axis of the VMO, and clearly visualizes fascicles on the U/S screen. VMO-ACSA was assessed at one fingerbreadth above the patella, upper border of patella and at mid-patellar level (level of femoral condyles). Three images were acquired and stored for offline analysis.

\section{Statistical analysis}

The statistical analyses were performed using IBM SPSS Statistics for Windows, Version 20.0 (IBM Co., Armonk, NY, USA). Descriptive statistics (mean and standard deviation [SD]) were used to describe the VMO muscle ACSA in the study groups. Differences between painless and painful knees in addition to differences between MRI and U/S measurements were assessed by two-way analysis of variance (ANOVA) with post-hoc Tukey tests. Furthermore, the difference \% in VMO-ACSA (in MRI measurements) for painful vs. painless knee was calculated and expressed [10]. Results were considered statistically significant when $P$-value was $<0.05$.
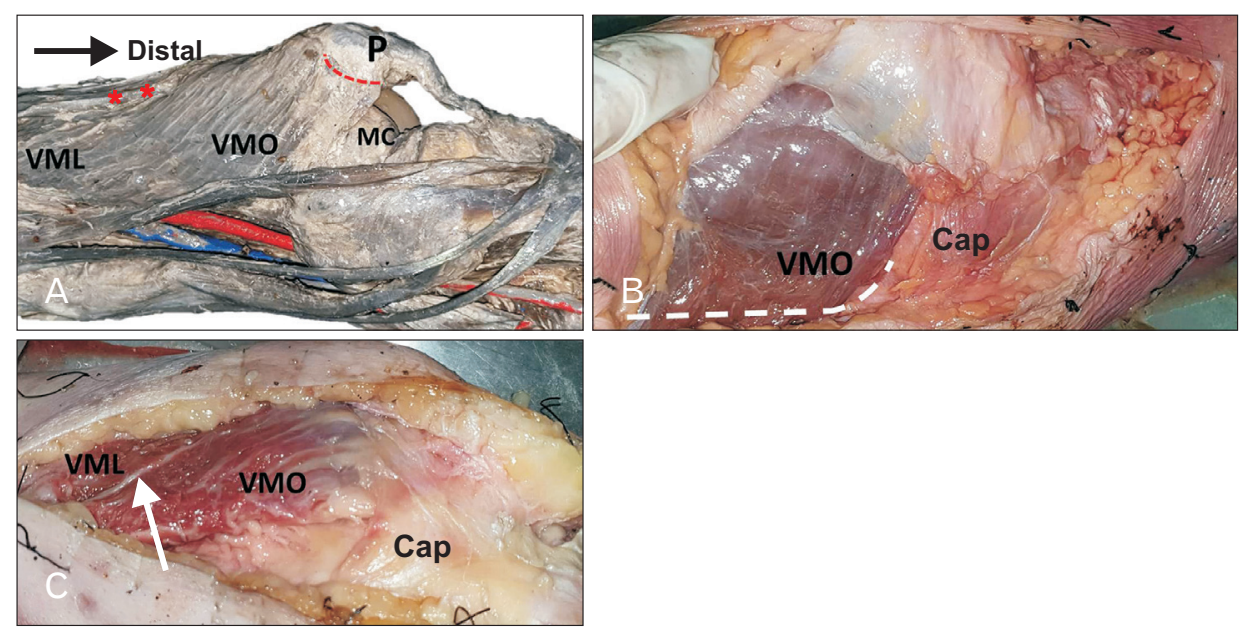

Fig. 1. VM muscle dissection showing (A) VML has vertical fibers and VMO with increased obliquity of muscle fibers. VMO inserted into the medial proximal margin of the patella with fleshy fibers (dotted red line). Lowermost fibers of VMO covered the medial aspect of medial condyle of femur. Nerve to VM runs in a separate fibrotic tunnel (red asterisks). (B) The dotted white line demonstrates the origin of VMO. VMO muscle fibers merge with fibrous capsule of the knee joint. (C) A fascial plane (arrow) separates the VM into two independent parts. Also, the VMO overlaps and merges with the joint capsule. cap, capsule of the knee joint; MC, medial condyle of femur; P, patella; VM, vastus medialis; VML, vastus medialis longus; $\mathrm{VMO}$, vastus medialis oblique. 


\section{Results}

\section{Origin, insertion, innervation and architecture of the VMO muscle}

VM has a proximal part called the vastus medialis longus (VML) that has vertical fibers. The VMO represents the distal portion of the VM and it is defined by an increased obliquity of the muscle fibers, which originate largely from the same inserting points of pubic part of adductor magnus, the medial lip of linea aspera (Fig. 1) and medial supracondylar line (Figs. 1, 2). The strong muscle belly of VMO finally inserted into the medial proximal margin of the patella and capsule of the knee joint.by fleshy fibers (Figs. 1-3). VMO further has a small region of direct continuity with the patellar tendon (Fig. 4). At the patellar insertion, VMO reached its maximal obliquity. Nine of the 10 limbs analyzed (90\%) exhibited a distinct change in muscle fiber direction in VMO. Separate innervation (i.e., a branch that was given off from the posterior division of the femoral nerve was observed in 9 specimens; the VM branch, coursing distally along the anteromedial border of the muscle in a separate fibrotic tunnel (Fig. 1). VMO showed a variable architecture. Seven specimens of the examined cadaveric lower extremities demonstrated fibro-fascial plane of epimysium that would clearly separate the two portions of the VM muscle from one another (Figs. 1, 3). Three specimens showed no separation of VM muscle into two independent parts. Through serial transverse cuts at lower third of femur, VMO covered the anteromedial surface of medial condyle of femur totally at epicondylar level; consequently, the knee joint space was compressed medially (Fig. 2). In addition, in MRI coronal sections of fully extended knee, the medial condyle was pushed posteriorly than the lateral condyle (Fig. 4). To illustrate the VMO muscle in sonogram, we used a diagram from Anatomage. The lower horizontal fibers of VMO were observed to insert in the medial margin of patella as it wraps
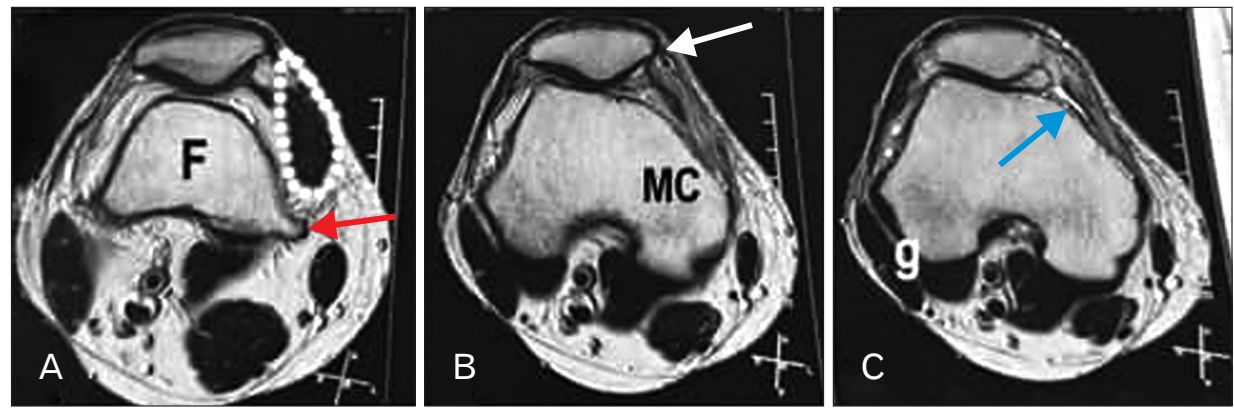

Fig. 2. MRI cross sections of the knee joint through the lower third of the right thigh showing: (A) at lower end of shaft of femur, VMO (marked by dotted white line) originates from medial supracondylar line (red arrow). (B) at medial epicondyle, VMO totally covered the medial aspect of medial condyle. VMO inserted in patella by fleshy fibers (white arrow) (C) at level of the popliteal groove, VMO was well defined that compressed the knee joint space (blue arrow) medially. F, lower end of shaft of femur; g, popliteal groove; MC, medial condyle of femur; MRI, magnetic resonance imaging; VMO, vastus medialis oblique.
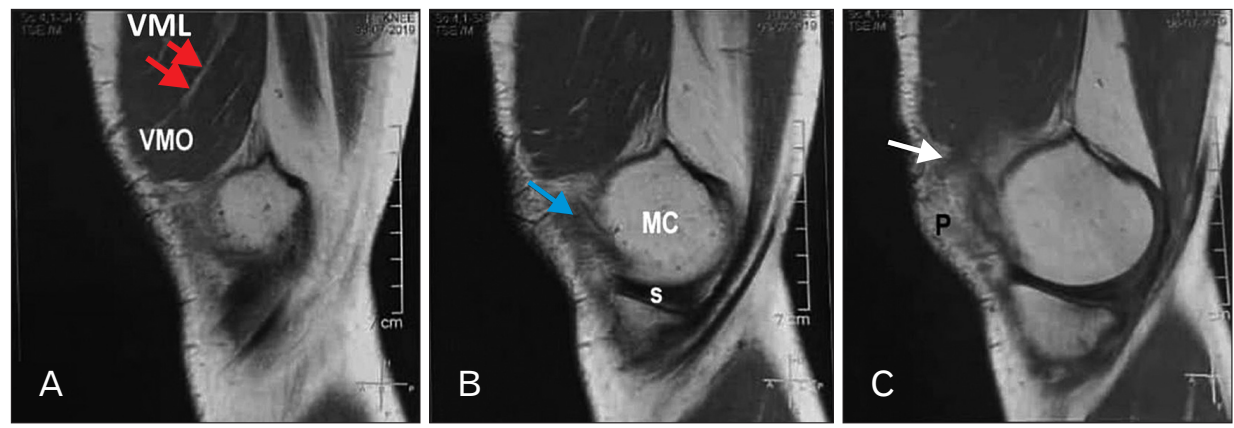

Fig. 3. MRI sagittal sections through the extensor apparatus of the knee showing: (A) a fascial plane (red arrows) separates VM into two parts. (B) Lowermost fibers of VMO (blue arrow) overlap the anterior aspect of medial condyle of femur. (C) VMO inserted into the medial proximal margin of the patella by fleshy fibers (white arrow). MC, medial condyle of femur; MRI, magnetic resonance imaging; P, patella; VM, vastus medialis; VML, vastus medialis longus; VMO, vastus medialis oblique. 

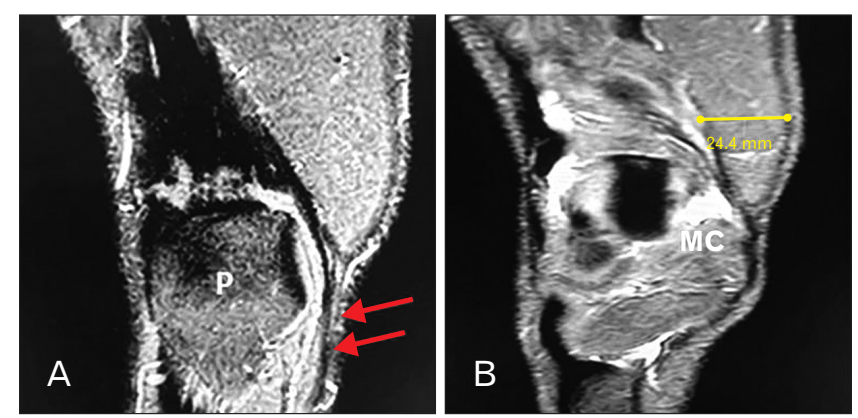

Fig. 4. MRI coronal sections through the extensor apparatus of the knee showing: (A) VMO inserted into the medial proximal margin of the patella and has a small region of direct continuity with the patellar tendon (red arrows) (B) Lowermost fibers of VMO overlapped the anterior aspect of the medial condyle of femur. In fully extended knee, the medial condyle was pushed posteriorly than the lateral condyle. MC, medial condyle of femur; MRI, magnetic resonance imaging; P, patella; VMO, vastus medialis oblique.
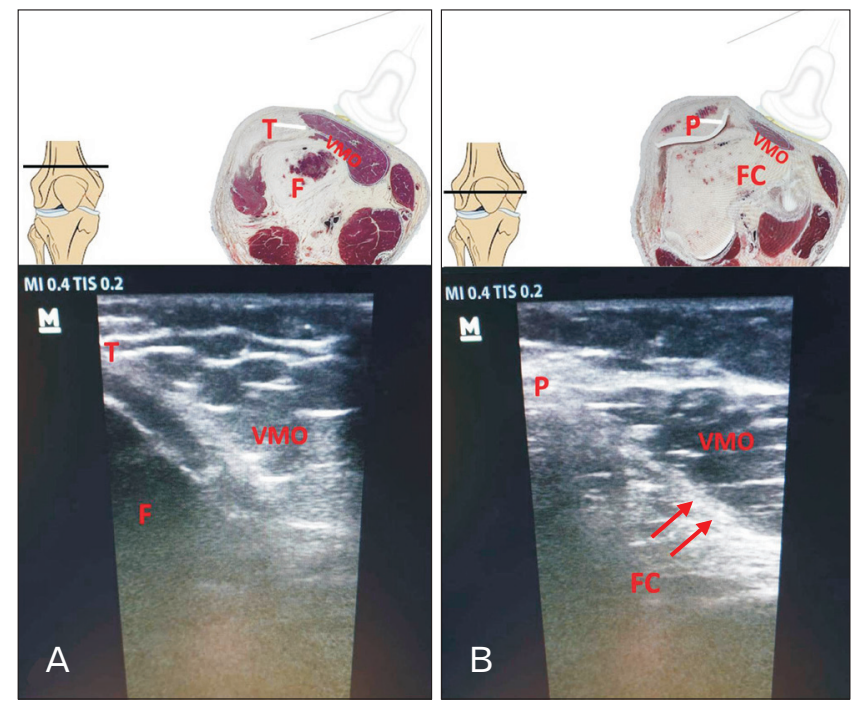

Fig. 5. U/S images of the VMO from a representative participant made by linear probe. (A) Crosssection showing anatomical structures immediately above the patella (B) Crosssection at midpatellar level. arrow, the VMO overlaps and merges with the joint capsule; F, femur; Fc, femoral condyle; P, patella; T, quadriceps tendon; U/S, ultrasound; VMO, vastus medialis oblique.

around the medial femoral condyle. VMO fascicles were transversely oriented near the point of insertion (Fig. 5).

\section{VMO anatomical cross-sectional area (ACSA) in painful vs. painless limbs}

The mean values of VMO-ACSA in painful vs. painless knees are summarized in Table 1. In painless knees, VMO cross sectional area was measured on MRI image from proximal to distal; at lower end of shaft of femur it was $23.2 \pm 1.9 \mathrm{~cm}^{2}$ (mean \pm SD), $16.7 \pm 1.7 \mathrm{~cm}^{2}$ at upper border of patella and, $14.1 \pm 1.1 \mathrm{~cm}^{2}$ at mid-patellar level (level of femoral condyles). However, no statistically significant difference was found between MRI and U/S measurements in the corresponding levels. In painful knee, all parameters were significantly decreased as compared to painless knee. The noticed decrease in VMO-ACSA in painful knee was expressed as a percentage decrease in relation to painless knee. The differences $\%$ in VMO-ACSA were calculated in painful knee vs. painless knee; $-17.2 \% \pm 11.0 \%$ at lower end of shaft of femur, $-21.1 \% \pm 6.0 \%$ at upper border of patella, $-36.7 \% \pm 11.0 \%$ at mid-patellar level.

\section{Discussion}

The quadriceps muscle is a primary contributor to both functional knee joint stability and knee joint loading [22]. Dynamic knee joint stability may be compromised when weak quadriceps muscles are unable to provide adequate 
Table 1. A comparison between vastus medialis oblique anatomical cross-sectional area $\left(\mathrm{cm}^{2}\right)$ measured by MRI and U/S in painless and painful knee

\begin{tabular}{|c|c|c|c|c|}
\hline Anatomical level & & Painless knee & Painful knee & $\begin{array}{c}\text { Difference \% in } \\
\text { MRI measurements } \\
\text { (painful vs. painless knee) }\end{array}$ \\
\hline \multirow[t]{2}{*}{ 1- Lower end of shaft of femur } & MRI measurement & $23.2 \pm 1.9$ & $19.2 \pm 1.6^{*}$ & $-17.2 \pm 11.0 \%$ \\
\hline & $\mathrm{U} / \mathrm{S}$ measurement & $22.5 \pm 0.58$ & $19.5 \pm 0.04^{*}$ & \\
\hline \multirow[t]{2}{*}{ 2- Upper border of patella } & MRI measurement & $16.7 \pm 1.7$ & $13.02 \pm 1.9^{*}$ & $-21.1 \pm 6.0 \%$ \\
\hline & $\mathrm{U} / \mathrm{S}$ measurement & $16.5 \pm 0.8$ & $12.7 \pm 0.62^{*}$ & \\
\hline \multirow[t]{2}{*}{ 3- Mid-patellar level } & MRI measurement & $14.1 \pm 1.1$ & $8.66 \pm 0.6^{*}$ & $-36.7 \pm 11.0 \%$ \\
\hline & U/S measurement & $13.5 \pm 1.14$ & $9.4 \pm 0.08^{*}$ & \\
\hline
\end{tabular}

Values are presented as mean $\pm \mathrm{SD}$.

MRI, magnetic resonance imaging; U/S, ultrasound.

${ }^{*} P<0.05$ is statistically significant as compared to painless knee at the same level.

control of tibial translation during ambulation, increasing risk of damage to joint structures [23, 24]. Kim et al. [25] found that with respect to knee extensor muscle strength, the more painful knees showed significantly lower strength than the less painful knees at angular velocities and there is a significant association between dynamic balance measurements and quadriceps strength at angular velocity. The current study provides an insight for the morphology of the $\mathrm{VMO}$ and its participation in anterior knee pain.

A closer look at the VM reveals that the uppermost fibers (VML) are more vertically oriented while the lower fibers (VMO) are more obliquely oriented [11]. VMO has received moderate attention as potentially being more responsible for medial tension on the patella.

Muscles with a larger number of insertion and origin points have a greater impact on the musculoskeletal system when perturbed than muscles with few insertion and origin points [26]. The present study showed that VMO originates from medial lip of linea aspera and has attachments to the medial supracondylar line as it wraps around the femur. VMO inserted directly to the upper interior corner of the patella by fleshy fibers. Such finding might reflect the force of movement (applied to the patella) produced by this muscle. Lefebvre et al. [9]; Grob et al. [17] and Grob et al. [27] have demonstrated similar attachment. Motor point stimulation and anatomical dissection clearly showed that VM distal fibers were attached directly to the medial aspect of the patella that contributes to dynamic medial patellar stabilization and opposing its lateral dislocation [28]. Altered muscle activity and weaknesses in VMO cause mal-tracking of the patella and subsequent damage to surrounding structures and aching pain [29]. In the current research, the oblique fibers of VM are not only involved in the medial border of the patella, but additionally, they have a minor area of direct continuity with the patellar tendon. Also, VMO muscle fibers merge with the knee joint capsule. These observations were in accordance with a previous study [17]. Recently, Castanov et al. [11] stated that VMO attached to the mid-portion of the patella, the medial patellofemoral ligament and adductor magnus tendon. Its attachment and oblique alignment provide a mechanical advantage to promote medial stabilizing force to the patella. Also, Muhamed et al. [30] reported that the patellar thickness is important variable to be considered, while assessing the South Indian female for patellofemoral disorders.

Some of the examined cadavers clearly had a nerve branch entering the distal portion of VM muscle separately from the proximal portion. Although most muscles are innervated from their deep surface, it is not unusual for a nerve to travel superficially in a fascial plane prior to entering a muscle belly. The branch to VM from femoral nerve was the furthermost distally situated, almost half-way down the thigh. The branch was well distinct running lengthwise the anteromedial surface of the muscle with the saphenous nerve and was possibly mobilized well proximally. The nerve to VM is designated as giving a short branch entering the muscle proximally, and a longer nerve fiber wandering in the adductor canal before inflowing the muscle belly in its mid-portion [31]. Our nerve branch corresponds to the longer medial branch noted by Page et al. [31]. Separate nerve innervation is a sufficient evidence to support the concept that VMO is an anatomically and functionally separate muscle from VML in the quadriceps femoris muscle group.

$\mathrm{VM}$ is more powerful and extends more distally than $\mathrm{VL}$ and its relative predominance is traditionally described as medial patellar stabilizer [10]. The findings of this study 
indicate that VMO is sufficient to rotate the medial condyle of femur internally and the muscle could act independently. "Proof of medial rotation" has been provided by VMO spiral fiber arrangement that covered the anteromedial aspect of the medial condyle. Moreover, the joint space is more compressed medially. Medial rotation of the femur (locking) occurs during the last 30 degrees of extension and locking is produced by the continued action of the same muscles that produce extension i.e., the quadriceps femoris muscle [32]. Moreover, Toumi et al. [33] stated that the medial fusing points of the quadriceps tendon in the intermediate layers violates the insertion of the VM with potential consequences on the terminal phase of extension. Recently, Chang et al. [34] recorded that an appropriate VMO: VL ratio had beneficial effects on PFPS.

Several different clinical tools have been utilized to objectively report the muscle tension in the clinical and operative settings. The use of MRI is a reasonable and more logical approach, but care must be taken to ensure VMO attachment to the patella is accurate. In the present study, the VMO-ACSA of painless knees was $23.2 \pm 1.9 \mathrm{~cm}^{2}$ at lower end of shaft of femur, $16.7 \pm 1.7 \mathrm{~cm}^{2}$ at medial epicondyle, $14.1 \pm 1.1 \mathrm{~cm}^{2}$ at popliteal groove with no statistically significant difference between MRI and U/S measurements. The relative ACSA taken by VMO varies substantially with anatomical location. In the current investigation, we made an attempt to overcome this limitation by selecting a variable slice level among the lower end of the femur. Sattler et al. [10] recorded that in the distal third of the quadriceps femoris muscle, VM took the largest (36\%) proportion of the total quadriceps volume. The relative contribution of VM to the total quadriceps increased from proximal to distal. A sum of researches have long-established the trustworthiness of U/S procedure for assessing the dimensions of the quadriceps muscle in health [35]. U/S has the benefit of being movable and encompasses no ionizing radiation and can conclude breadth and cross-sectional areas of superficial muscles. It is potential to quantity basic parameters of muscle construction with $\mathrm{U} / \mathrm{S}$ investigation, like muscle volume, fascicle length (muscle fiber length that indicates the variety of lengths over which the muscle is capable of actively producing force), and pennation angle (muscle fiber angle comparative to the force-generating alliance) [36].

ACSA was used to represent muscle size as it has the benefit of being a good predictor of both isometric and isokinetic muscle force $[37,38]$. In the current work, the VMO-ACSA decreased significantly and similarly in all the experimen- tal groups comparing painless to painful knee (decrements ranged from $17.2 \%$ to $36.7 \%$ ). Painful limbs displayed significantly smaller VMO-ACSA. Therefore, impaired VMO muscle function may lead to injurious loading of articular structures, including menisci, ligaments, cartilage, and bone. Wang et al. [39] reported that a low VM-ACSA was associated with pain and with medial tibial cartilage volume loss. Providing support for this hypothesis are data from human studies in which quadriceps muscle dysfunction may precede degenerative changes [40]. Patients with PFPS were found to have a lower muscle activity ratio between VM and VL muscles than healthy subjects [29]. Engelina et al. [2] added that VMO angle may be a contributory factor in a subset of PFPS, especially in patients who have patellar instability. Previous studies have reported a reduction in anterior knee pain following an increase in muscle strength with exercise training in people with symptomatic knee osteoarthritis [41, 42]. Further, significant increase in VM-ACSA in response to training over time was found to be associated with a concurrent reduction in anterior knee pain, a reduced medial tibial cartilage loss, and a reduced danger of performing knee replacement [39]. So, the current study supports training intervention as a successful measure to induce VMO muscle strength, even at advanced age. This may effectively increase joint health and protect against knee degenerative changes.

Changes in VMO-ACSA as a percentage over a certain time duration using $U / S$ is not widely used for screening and staging. In the near future, it may become a valid method to assess muscle in different settings. Also, further study is recommended to explore the differences in VMO-ACSA as regards age, sex and differences in body weight and to assess whether these correlate to functional findings.

In conclusion, VMO is a distinct muscle within the quadriceps femoris group. VMO would track the patella medially and participating in last phase of knee extension. The assessment of the VMO-ACSA by ultrasonography may constitute promising and reliable tool to evaluate PFPS staging.

\section{ORCID}

Marwa M El Sawy:

https://orcid.org/0000-0003-4459-6216

Dalia M E EL Mikkawy:

https://orcid.org/0000-0001-5101-9797

Sayed M El-Sayed: https://orcid.org/0000-0002-9025-4690

Ahmed M. Desouky: 
https://orcid.org/0000-0001-7962-5086

\section{Author Contributions}

Conceptualization: MMES. Data acquisition: AMD. Data analysis or interpretation: MMES, SMES. Drafting of the manuscript: DMEEM, AMD. Critical revision of the manuscript: MMES, DMEEM, SMES, AMD. Approval of the final version of the manuscript: all authors.

\section{Conflicts of Interest}

No potential conflict of interest relevant to this article was reported.

\section{Acknowledgements}

The authors would like to extend their appreciation to team of Anatomage coordinating center at anatomy department, Faculty of medicine, Ain Shams University for supporting this research. Also, we would like to thank the participants, the study investigators and the staff at the clinical centers for generating the image data sets used in this analysis.

\section{References}

1. Myer GD, Ford KR, Barber Foss KD, Goodman A, Ceasar A, Rauh MJ, Divine JG, Hewett TE. The incidence and potential pathomechanics of patellofemoral pain in female athletes. Clin Biomech (Bristol, Avon) 2010;25:700-7.

2. Engelina S, Antonios T, Robertson CJ, Killingback A, Adds PJ. Ultrasound investigation of vastus medialis oblique muscle architecture: an in vivo study. Clin Anat 2014;27:1076-84.

3. Arazpour M, Bahramian F, Abutorabi A, Nourbakhsh ST, Alidousti A, Aslani $\mathrm{H}$. The effect of patellofemoral pain syndrome on gait parameters: a literature review. Arch Bone Jt Surg 2016;4:298-306

4. Arazpour M, Notarki TT, Salimi A, Bani MA, Nabavi H, Hutchins SW. The effect of patellofemoral bracing on walking in individuals with patellofemoral pain syndrome. Prosthet Orthot Int 2013;37:465-70.

5. Johnston LB, Gross MT. Effects of foot orthoses on quality of life for individuals with patellofemoral pain syndrome. J Orthop Sports Phys Ther 2004;34:440-8.

6. Dixit S, DiFiori JP, Burton M, Mines B. Management of patellofemoral pain syndrome. Am Fam Physician 2007;75:194-202.

7. Murphy AC, Muldoon SF, Baker D, Lastowka A, Bennett B, Yang M, Bassett DS. Structure, function, and control of the hu- man musculoskeletal network. PLoS Biol 2018;16:e2002811.

8. Hortobágyi T, Garry J, Holbert D, Devita P. Aberrations in the control of quadriceps muscle force in patients with knee osteoarthritis. Arthritis Rheum 2004;51:562-9.

9. Lefebvre R, Leroux A, Poumarat G, Galtier B, Guillot M, Vanneuville G, Boucher JP. Vastus medialis: anatomical and functional considerations and implications based upon human and cadaveric studies. J Manipulative Physiol Ther 2006;29:139-44.

10. Sattler M, Dannhauer T, Ring-Dimitriou S, Sänger AM, Wirth W, Hudelmaier M, Eckstein F. Relative distribution of quadriceps head anatomical cross-sectional areas and volumes-sensitivity to pain and to training intervention. Ann Anat 2014;196:464-70.

11. Castanov V, Hassan SA, Shakeri S, Vienneau M, Zabjek K, Richardson D, McKee NH, Agur AMR. Muscle architecture of vastus medialis obliquus and longus and its functional implications: a three-dimensional investigation. Clin Anat 2019;32:515-23

12. Sattler M, Dannhauer T, Hudelmaier M, Wirth W, Sänger AM, Kwoh CK, Hunter DJ, Eckstein F. Side differences of thigh muscle cross-sectional areas and maximal isometric muscle force in bilateral knees with the same radiographic disease stage, but unilateral frequent pain- data from the osteoarthritis initiative. Osteoarthritis Cartilage 2012;20:532-40.

13. Glass NA, Torner JC, Frey Law LA, Wang K, Yang T, Nevitt MC, Felson DT, Lewis CE, Segal NA. The relationship between quadriceps muscle weakness and worsening of knee pain in the MOST cohort: a 5-year longitudinal study. Osteoarthritis Cartilage 2013;21:1154-9.

14. Villafañe JH, Bissolotti L, La Touche R, Pedersini P, Negrini S. Effect of muscle strengthening on perceived pain and static knee angles in young subjects with patellofemoral pain syndrome. J Exerc Rehabil 2019;15:454-9.

15. Abe T, Loenneke JP, Thiebaud RS. Morphological and functional relationships with ultrasound measured muscle thickness of the lower extremity: a brief review. Ultrasound 2015;23:166-73

16. Parry SM, El-Ansary D, Cartwright MS, Sarwal A, Berney S, Koopman R, Annoni R, Puthucheary Z, Gordon IR, Morris PE, Denehy L. Ultrasonography in the intensive care setting can be used to detect changes in the quality and quantity of muscle and is related to muscle strength and function. J Crit Care 2015;30:1151.e9-14.

17. Grob K, Manestar M, Filgueira L, Ackland T, Gilbey H, Kuster MS. New insight in the architecture of the quadriceps tendon. J Exp Orthop 2016;3:32.

18. Ayala-Mejias JD, Garcia-Gonzalez B, Alcocer-Perez-España L, Villafañe JH, Berjano P. Relationship between widening and position of the tunnels and clinical results of anterior cruciate ligament reconstruction to knee osteoarthritis: 30 patients at a minimum follow-up of 10 years. J Knee Surg 2017;30:501-8.

19. Ema R, Wakahara T, Hirayama K, Kawakami Y. Effect of knee alignment on the quadriceps femoris muscularity: crosssectional comparison of trained versus untrained individuals 
in both sexes. PLoS One 2017;12:e0183148.

20. Rueden CT, Schindelin J, Hiner MC, DeZonia BE, Walter AE, Arena ET, Eliceiri KW. ImageJ2: ImageJ for the next generation of scientific image data. BMC Bioinformatics 2017;18:529.

21. Franchi MV, Longo S, Mallinson J, Quinlan JI, Taylor T, Greenhaff PL, Narici MV. Muscle thickness correlates to muscle cross-sectional area in the assessment of strength traininginduced hypertrophy. Scand J Med Sci Sports 2018;28:846-53.

22. Winby CR, Lloyd DG, Besier TF, Kirk TB. Muscle and external load contribution to knee joint contact loads during normal gait. J Biomech 2009;42:2294-300.

23. Andriacchi TP, Koo S, Scanlan SF. Gait mechanics influence healthy cartilage morphology and osteoarthritis of the knee. J Bone Joint Surg Am 2009;91(Suppl 1):95-101.

24. Englund M. The role of biomechanics in the initiation and progression of OA of the knee. Best Pract Res Clin Rheumatol 2010;24:39-46.

25. Kim D, Park G, Kuo LT, Park W. The effects of pain on quadriceps strength, joint proprioception and dynamic balance among women aged 65 to 75 years with knee osteoarthritis. BMC Geriatr 2018;18:245.

26. Chopp-Hurley JN, Langenderfer JE, Dickerson CR. Probabilistic evaluation of predicted force sensitivity to muscle attachment and glenohumeral stability uncertainty. Ann Biomed Eng 2014;42:1867-79.

27. Grob K, Manestar M, Filgueira L, Kuster MS, Gilbey H, Ackland $\mathrm{T}$. The interaction between the vastus medialis and vastus intermedius and its influence on the extensor apparatus of the knee joint. Knee Surg Sports Traumatol Arthrosc 2018;26:72738.

28. Belli G, Vitali L, Botteghi M, Vittori LN, Petracci E, Latessa PM. Electromyographic analysis of leg extension exercise during different ankle and knee positions. J Mech Med Biol 2015;15:1540037.

29. Kang JI, Park JS, Choi H, Jeong DK, Kwon HM, Moon YJ. A study on muscle activity and ratio of the knee extensor depending on the types of squat exercise. J Phys Ther Sci 2017;29:43-7.

30. Muhamed R, Saralaya VV, Murlimanju BV, Chettiar GK. In vivo magnetic resonance imaging morphometry of the patella bone in South Indian population. Anat Cell Biol 2017;50:99103.

31. Page BJ, Mrowczynski OD, Payne RA, Tilden SE, Lopez H, Rizk E, Harbaugh K. The relative location of the major femoral nerve motor branches in the thigh. Cureus 2019;11:e3882.

32. Chaitow L, DeLany J. Clinical application of neuromuscular techniques volume 2, the lower body. 2nd ed. Edinburgh: Churchill Livingstone; 2011.

33. Toumi H, Poumarat G, Benjamin M, Best TM, F'Guyer S, Fairclough J. New insights into the function of the vastus medialis with clinical implications. Med Sci Sports Exerc 2007;39:11539.

34. Chang WD, Huang WS, Lai PT. Muscle activation of vastus medialis oblique and vastus lateralis in sling-based exercises in patients with patellofemoral pain syndrome: a cross-over study. Evid Based Complement Alternat Med 2015;2015:740315.

35. Thomaes T, Thomis M, Onkelinx S, Coudyzer W, Cornelissen V, Vanhees L. Reliability and validity of the ultrasound technique to measure the rectus femoris muscle diameter in older CAD-patients. BMC Med Imaging 2012;12:7.

36. Stevens DE, Smith CB, Harwood B, Rice CL. In vivo measurement of fascicle length and pennation of the human anconeus muscle at several elbow joint angles. J Anat 2014;225:502-9.

37. Blazevich AJ, Coleman DR, Horne S, Cannavan D. Anatomical predictors of maximum isometric and concentric knee extensor moment. Eur J Appl Physiol 2009;105:869-78.

38. Trezise J, Blazevich AJ. Anatomical and neuromuscular determinants of strength change in previously untrained men following heavy strength training. Front Physiol 2019;10:1001.

39. Wang Y, Wluka AE, Berry PA, Siew T, Teichtahl AJ, Urquhart DM, Lloyd DG, Jones G, Cicuttini FM. Increase in vastus medialis cross-sectional area is associated with reduced pain, cartilage loss, and joint replacement risk in knee osteoarthritis. Arthritis Rheum 2012;64:3917-25.

40. Miyazaki T, Wada M, Kawahara H, Sato M, Baba H, Shimada S. Dynamic load at baseline can predict radiographic disease progression in medial compartment knee osteoarthritis. Ann Rheum Dis 2002;61:617-22.

41. Roos EM, Herzog W, Block JA, Bennell KL. Muscle weakness, afferent sensory dysfunction and exercise in knee osteoarthritis. Nat Rev Rheumatol 2011;7:57-63.

42. Ruhdorfer A, Dannhauer T, Wirth W, Hitzl W, Kwoh CK, Guermazi A, Hunter DJ, Benichou O, Eckstein F. Thigh muscle cross-sectional areas and strength in advanced versus early painful osteoarthritis: an exploratory between-knee, withinperson comparison in osteoarthritis initiative participants. Arthritis Care Res (Hoboken) 2013;65:1034-42. 